\title{
Classification of operator algebraic conformal field theories
}

\author{
YASUYUKI KAWAHIGASHI * \\ Department of Mathematical Sciences \\ University of Tokyo, Komaba, Tokyo, 153-8914, Japan \\ e-mail: yasuyuki@ms.u-tokyo.ac.jp
}

\begin{abstract}
We give an exposition on the current status of classification of operator algebraic conformal field theories. We explain roles of complete rationality and $\alpha$-induction for nets of subfactors in such a classification and present the current classification result, a joint work with R. Longo, for the case with central charge less than 1 , where we have a complete classification list consisting of the Virasoro nets, their simple current extensions of index 2, and four exceptionals. Two of the four exceptionals appear to be new.
\end{abstract}

\section{Introduction}

Classification of conformal field theory is obviously one of the most important and exciting problems in mathematical physics today. Here we review the current status of the classification theory of 1-dimensional operator algebraic conformal field theories.

First, we explain what we mean by "1-dimensional operator algebraic conformal field theories" and how they naturally appear in the setting of $1+1$-dimensional conformal field theory. We are now in the framework of algebraic quantum field theory in the sense of [21]. In the algebraic quantum field theory, we consider a net of von Neumann algebras $\{\mathcal{B}(O)\}$ where $O$ is a certain bounded region in the Minkowski space, and the physical idea is that the von Neumann algebra $\mathcal{B}(O)$ is generated by physical quantities observable in the region $O$. From a physical viewpoint, the most natural setting is that we have a 4 -dimensional Minkowski space and $O$ is a double cone, which is defined to be a set of the form $\left(x+V_{+}\right) \cap\left(y+V_{-}\right)$, where

$$
V_{ \pm}=\left\{z=\left(z_{0}, z_{1}, z_{2}, z_{3}\right) \in \mathbb{R}^{4} \mid z_{0}^{2}-z_{1}^{2}-z_{2}^{2}-z_{3}^{2}>0, \pm z_{0}>0\right\} .
$$

We say a net because the index set of double cones is directed with respect to inclusions. Then we impose some physically natural set of axioms on the net $\mathcal{B}$, as

${ }^{*}$ The author was supported in part by JSPS Grant. 
explained below. So mathematically speaking, this is a study of a family of von Neumann algebras (on a fixed Hilbert space) parametrized by double cones subject to certain set of axioms. Although the 4-dimensional Minkowski space seems most natural at first sight, it has been known that a net on the 2-dimensional Minkowski space produces interesting structures both from physical and mathematical viewpoints. Such a net is called a two-dimensional net. Using a new set of coordinates $\left(x_{0}+x_{1}, x_{0}-x_{1}\right)$ in the two-dimensional Minkowski space, a double cone $O$ becomes a product of two intervals $I=(a, b), J=(c, d)$. We now start with a net $\mathcal{B}$ of von Neumann algebras on the two-dimensional Minkowski space. Then as in [36], we obtain a decomposition $\mathcal{A}_{L}(I) \otimes \mathcal{A}_{R}(J) \subset \mathcal{B}(I \times J)$, where $\mathcal{A}_{L}, \mathcal{A}_{R}$ are nets of von Neumann algebras on one-dimensional spaces. Such one-dimensional nets are called chiral theories. In this way, we are naturally led to a study of one-dimensional nets of von Neumann algebras, starting from a study of two-dimensional nets of von Neumann algebras. As a part of the set of axioms for algebraic quantum field theory, we assume that we have a certain group representing space-time symmetry and its projective unitary representation on the Hilbert space on which our net of von Neumann algebras act. We require compatibility of this projective unitary representation and the net of von Neumann algebras in an appropriate sense and this compatibility condition is called covariance. A space-time symmetry of the two-dimensional Minkowski space passes to a symmetry of the one-dimensional space in the above decomposition, so we now consider a symmetry group of the one-dimensional space. It is often more convenient to compactify the one-dimensional space and use $S^{1}$ rather than $\mathbb{R}$, and we do so here. There can be more than one choice for such a symmetry group of $S^{1}$, and we often use the name "conformal field theory" when we choose the Möbius group $P S L(2, \mathbb{R})$ as the symmetry group. In this paper, we use this Möbius group, or even more strongly, the diffeomorphism group Diff $\left(S^{1}\right)$ later. (See [24] for a precise definition of the diffeomorphism covariance.) Thus our set of axioms for one-dimensional net of von Neumann algebras is now described as follows.

By an interval, we mean a non-empty, non-dense, open, and connected set in $S^{1}$. We study a family of von Neumann algebras $\mathcal{A}(I)$ on a fixed Hilbert space $H$ parameterized by intervals $I$. (Now the set of intervals is not directed with respect to inclusions, so the terminology "net" is not appropriate. The name "precosheaf" is also often used instead, because of this reason, but the name "net" has been used in many cases, and we also use it here.) Our set of axioms is as follows. (See [19, 24] for precise descriptions of these properties.)

\section{Isotony}

2. Locality

3. Covariance

4. Positivity of the energy

5. Unique existence of a vacuum vector 
Such a family of von Neumann algebras is also called a local conformal net. Then the Haag duality, $\mathcal{A}\left(I^{\prime}\right)=\mathcal{A}(I)^{\prime}$, automatically holds, where the prime on the left hand side means the interior of the complement of the interval $I$, and the prime on the right hand side denotes the commutant of $\mathcal{A}(I)$.

The main object of mathematical studies of such nets is their classification. By a "classification", we mean obtaining a complete invariant up to isomorphism and a complete list of all the isomorphism classes. Both problems are very difficult, but we explain the first step to these problems here. The invariant to the classification problem we now use is a representation theory of a net. Under the above set of axioms and the split property, which is often assumed as an additional axiom, each von Neumann algebra $\mathcal{A}(I)$ is an injective type $\mathrm{III}_{1}$ factor. In general, all representations of a type III factor on separable Hilbert spaces are unitarily equivalent, so representation theory for a single algebra $\mathcal{A}(I)$ is trivial. However, we obtain non-trivial and useful information by considering representations of a family of type III factors. That is, we consider a family of representations $\pi_{I}$ of $\mathcal{A}(I)$ on one Hilbert space parameterized by intervals $I$ on the circle, with a natural compatibility condition and a covariance property with respect to the symmetry group. (By [18], a covariance property with respect to the Möbius group automatically holds, if we have strong additivity and the statistical dimension, which is explained below, of the representation is finite.) By adapting the standard Doplicher-Haag-Roberts (DHR) theory [11] to our onedimensional setting, we get a category of representations and each representation is realized as a DHR endomorphism. Then we have a notion of irreducibility, direct sums, irreducible decomposition, a statistical dimension, conjugates, unitary equivalence and a tensor product. It behaves like a category of unitary representations of a compact group. Here a statistical dimension plays a role of a usual dimension, but the values are now in the set of positive real numbers. A tensor product is given by a composition of DHR endomorphisms. In this way, we obtain a strict $C^{*}$-tensor category with conjugates, subobjects, and direct sums in the sense of [12,31].

In operator algebra theory, we often have an algebraic invariant from a certain representation theoretic consideration, and it becomes a complete invariant under an extra assumption of amenability in some sense, though proving completeness of such an invariant requires very deep analytic arguments. Also in this setting of algebraic quantum field theory, we expect that a representation category, possibly with some additional related data, should give a complete invariant of local conformal nets with some kind of amenability assumption, but results of such a general type have not been obtained and seem very difficult to prove. In this paper, we explain the first classification result [24] in this direction under an extra assumption on a central charge, a numerical invariant of a net that is also "representation theoretic".

When we assume covariance with respect to $\operatorname{Diff}\left(S^{1}\right)$ rather than $\operatorname{PSL}(2, \mathbb{R})$ for a local conformal net $\mathcal{A}$, we can define a central charge $c$ for $\mathcal{A}$ as follows. The corresponding infinite dimensional Lie algebra of the infinite dimensional Lie group $\operatorname{Diff}\left(S^{1}\right)$ is the celebrated Virasoro algebra, which is generated by $\left\{L_{m}\right\}_{m \in \mathbb{Z}}$ and a 
central element $c$ subject to the following relations.

$$
\left[L_{m}, L_{n}\right]=(m-n) L_{m+n}+\frac{c}{12}\left(m^{3}-m\right) \delta_{m,-n}
$$

where $m, n \in \mathbb{Z}$. In our setting, we obtain a representation of the Virasoro algebra from a local conformal net with diffeomorphism covariance, and we can define the central charge $c$, which is a numerical value. We then define this value to be the central charge of the net $\mathcal{A}$. It is clearly a numerical invariant of $\mathcal{A}$. It has been shown by Friedan-Qiu-Shenker [15] that this central charge value is in

$$
\{1-6 / m(m+1) \mid m=2,3,4, \ldots\} \cup[1, \infty)
$$

and the values $\{1-6 / m(m+1) \mid m=2,3,4, \ldots\}$ have been realized by GoddardKent-Olive [17]. (The values in $[1, \infty)$ are easier to realize.) Similarity of this restriction of possible values to the restriction of the values of the Jones indices of subfactors in [23] has been pointed by Jones himself since early days of subfactor theory. Furthermore, in subfactor theory, we have classification of paragroups of index less than 4 with the Dynkin diagrams $A_{n}, D_{2 n}, E_{6}, E_{8}$ by Ocneanu [32], which produces a classification of subfactors of the hyperfinite $\mathrm{II}_{1}$ factor with index less than 4 by Popa's analytic classification result [34]. (See the book [13] for details on this topic.) Also in the theory of Virasoro algebra, we have a classification involving $A-D-E$ Dynkin diagrams, that is, classification of modular invariants by Cappelli-Itzykson-Zuber [9]. (See also the book [10] for more on modular invariants.) The similarity between the two classification is lised in Table 1.

\begin{tabular}{|c|c|}
\hline Virasoro algebra & Subfactors \\
\hline central charge $c$ & Jones index \\
$c<1 \Rightarrow c=1-6 / m(m+1)[15]$ & index $<4 \Rightarrow$ index $=4 \cos ^{2} \pi / m[23]$ \\
\hline $\begin{array}{c}\text { classification of modular invariants }[9] \\
\text { with pairs of } A \text { - } D \text { - } E \text { diagrams }\end{array}$ & classification of paragroups $[32]$ \\
\hline
\end{tabular}

Table 1: Similarity between Virasoro algebra and subfactors

However, a real relation beyond formal similarity of the two classification theories was unknown. Furthermore, even on a formal level of similarity, it is not clear at all why we have pairs of Dynkin diagrams for Virasoro algebra and single Dynkin diagrams for subfactors. Our results in [24] on classification of local conformal nets, with diffeomorphism covariance and central charge less than 1 , give unification of these two classification theories.

We also remark a relation between such a study of local conformal nets and $E_{0^{-}}$ semigroups. The $E_{0}$-semigroups appearing here are not those of $B(H)$, but of the injective type $\mathrm{III}_{1}$-factor. Consider an inclusion $N \subset M$ of von Neumman algebra with a common cyclic separating vector $\Omega$. We have a modular automorphism group $\sigma_{t}^{M}$ with respect to the pair $(M, \Omega)$. Wiesbrock studied "half-sided modular inclusions" where we have $\sigma_{t}^{M}(N) \subset N$ for $t \leq 0$. This gives an $E_{0}$-semigroup of $N$, 
which is typically an injective type $\mathrm{III}_{1}$-factor in the setting appearing from a local conformal net. Wiesbrock [39] and Guido-Longo-Wiesbrock [20, Theorem 1.2] have shown that we have a triple of half-sided modular inclusions from a local conformal net on the circle, by splitting the circle into three intervals after removing three distinct points, and we can also recover the local conformal net from such a triple. In this sense, study of local conformal nets on the circle is "translated" to that of special type of $E_{0}$-semigroups of the injective type $\mathrm{III}_{1}$-factor.

\section{Completely rational nets and $\alpha$-induction}

We have mentioned that the invariant of a local conformal net we study is its representation theory, but in general, it is difficult to control representation theory of a net. A set of additional axioms has been proposed by us [25] in order to obtain a "good" representation theory as follows. (See [25] for the precise definitions.)

1. Split property

2. Strong additivity

3. Finite index condition

The first two conditions are rather well-known in algebraic quantum field theory. The definition of the new third condition, finite index condition, is as follows. Remove four distinct points from the circle $S^{1}$ and label the remaining four connected components as $I_{1}, I_{2}, I_{3}, I_{4}$ in a counterclockwise order. By locality, we have an inclusion $\mathcal{A}\left(I_{1}\right) \vee \mathcal{A}\left(I_{3}\right) \subset\left(\mathcal{A}\left(I_{2}\right) \vee \mathcal{A}\left(I_{4}\right)\right)^{\prime}$ of von Neumann algebras, which are now automatically injective type III-factors. The finite index condition means that the index of this subfactor is finite. (This condition is independent of choices of the four intervals.) When a local conformal net satisfies these three conditions, we say that it is completely rational. We have shown in [25] that if a local conformal net is completely rational, then we have only finitely many mutually inequivalent irreducible representations of the net. (Such finiteness is often called rationality. Our conditions are called complete rationality since they imply this rationality.) Furthermore, a weighted counting of the number of such representations, where each such representation is counted as the square of its statistical dimension, gives the index in the third condition above [25]. Furthermore, it has been known [14] that we have a braiding on the tensor category of representations of local conformal nets, and a notion of non-degeneracy of a braiding has been introduced in [35]. We have also shown in [25] that the representation category of a completely rational local conformal net has a non-degenerate braiding. In other words, the tensor category is modular in the sense of [37], and we have a unitary representation of the modular group $S L(2, \mathbb{Z})$ whose dimension is the number of unitary equivalence classes of irreducible representations of the net.

It is difficult in general to show that a certain local conformal net is completely rational. $\mathrm{F} . \mathrm{Xu}[42]$ proved the finiteness of the index, the third condition above, 
for the $S U(N)_{k}$-nets constructed by A. Wassermann [38] and thus proved their complete rationality. Complete rationality for orbifold and coset constructions has been also studied by $\mathrm{F}$. Xu [43, 44, 45], where many interesting properties and examples are presented. (Also see [29] in the case of the coset construction.) This complete rationality plays a role of amenability condition for local conformal nets.

In the classical representation theory, the notion of induced representation is very important and useful. Suppose we have a subgroup $H$ of $G$ and a unitary representation of $H$. Under a very mild condition, we can produce the induced representation of $G$ from a given representation of $H$. We would like to perform a similar construction for representation of local conformal nets. Suppose we have a net of subfactors $\mathcal{A} \subset \mathcal{B}$ on the circle. That is, this is a family of subfactors $\mathcal{A}(I) \subset \mathcal{B}(I)$ parametrized intervals $I \subset S^{1}$ with a certain natural set of axioms. (See [30,29] for a precise set of axioms.) We assume finiteness of the index $\mathcal{A}(I) \subset \mathcal{B}(I)$, which is independent of the interval $I$. Then, roughly speaking, we can produce a representation of the larger net $\mathcal{B}$ from a representation of the smaller net $\mathcal{A}$. This construction was introduced by Longo-Rehren [30] based on an old suggestion of J. Roberts, and many interesting properties and examples were studied by F. Xu [40, 41]. Further studies and applications have been made in $[1,2,3,4,5]$ under the name $\alpha$-induction. (This $\alpha$-induction also generalizes Ocneanu's graphical construction [33] for the Dynkin diagrams.) However, we do have three substantial differences between the induction for group representations and $\alpha$-induction for nets as follows. First, for groups $H \subset G$, the larger group has a larger symmetry, but the situation is opposite for nets. That is, the representation category of the larger group $G$ is larger, in an appropriate sense, than that of $H$, but the representation category of the larger net $\mathcal{B}$ is smaller than that of $\mathcal{A}$. The second difference is that $\alpha$-induction depends on the braiding structure of the representation category of the smaller net $\mathcal{A}$. A braiding always comes in a pair, a positive braiding and a negative braiding. (Graphically, they are represented by overcrossing and under-crossing, respectively.) We distinguish the two $\alpha$-inductions by the notations $\alpha^{+}$and $\alpha^{-}$. The third difference is that $\alpha$-induction does not produce a genuine representation of the larger net and it gives only a "fake representation", which is often called a soliton sector, although it behaves like a genuine representation in many senses. For example, it does have notions of a tensor product and a statistical dimension, but it does not have a braiding. (See [27] for a more general setting of such a tensor category.) Roughly speaking, the representation category of the larger net $\mathcal{B}$ is "too small" to accept the image of $\alpha$-induction. Actually, the intersection of the set of irreducible representations arising from a positive $\alpha$-induction and that from a negative $\alpha$-induction precisely gives the set of irreducible representations of the larger net $\mathcal{B}$. These differences produce a new interesting structure that does not appear in the classical setting of group representations, as explained in the next section.

We remark that we also have a machinery of restriction of a representation of a larger net to that of a smaller net, just as in the case of group representation, but $\alpha$-induction is much more useful than the restriction in many cases. One reason is 
that we often have a situation where we have enough information about the smaller nets and would like to obtain information on the larger nets.

\section{Modular invariants and classification of CFT}

Consider a net of subfactors $\mathcal{A} \subset \mathcal{B}$ with finite index as above, where $\mathcal{A}$ is completely rational. Choose irreducible representations $\lambda, \mu$ of the smaller net $\mathcal{A}$ and apply $\alpha^{ \pm}$-induction to them. Let $Z_{\lambda \mu}$ be $\operatorname{dim} \operatorname{Hom}\left(\alpha_{\lambda}^{+}, \alpha_{\mu}^{-}\right)$. Roughly speaking, this is a counting of the intersection of $\alpha_{\lambda}^{+}$and $\alpha_{\mu}^{-}$, and such an intersection occurs only in the genuine representation category of the larger net $\mathcal{B}$, as explained above. This number $Z_{\lambda \mu}$ is independent of choices of irreducible representations within unitary equivalence classes, of course, so in this way, we obtain a matrix $Z=\left(Z_{\lambda \mu}\right)$ of finite size whose rows and columns are indexed by unitary equivalences classes of irreducible representations of the net $\mathcal{A}$. The unitary representation of $S L(2, \mathbb{Z})$ mentioned above also has the same set of indices and we have proved in [3, Corollary 5.8] that this matrix $Z$ is in the commutant of the image of this unitary representation. The entries of the matrix $Z$ are obviously non-negative integers and we have $Z_{00}=1$, where 0 denotes the vacuum representation of the net $\mathcal{A}$. Such a matrix of non-negative integers in the commutant of a unitary representation of $S L(2, \mathbb{Z})$, subject to the normalization $Z_{00}=1$, is called a modular invariant of the unitary representation (or of the representation category of the net $\mathcal{A}$ ). In general, the unitary representation is not irreducible, so we can have something non-trivial in the commutant, but this unitary representation is often almost irreducible in some sense, and the commutant is quite small. So the modular invariant condition is very restrictive. In general, the number of modular invariants for a given unitary representation is always finite, and this finite number is often very small, such as 1,2 , or 3 , in a typical situation. The most famous classification list of such modular invariants has been obtained by Cappelli-Itzykson-Zuber [9] for the representation category of the $S U(2)_{k}$-net. In this case, we have at most three modular invariants for a given $k$. In principle, the classification problem of a given unitary representation of $S L(2, \mathbb{Z})$ is a problem in linear algebra and should be "solvable", but the classification can be very hard combinatorially, in practice. Gannon's series of papers deal with this problem. (See [16], for example.)

Our idea [24] for classification of local conformal nets is using such a classification of modular invariants through $\alpha$-induction. This is in the spirit of [6]. That is, start with a local conformal net $\mathcal{B}$ with diffeomorphism covariance. Then the algebras generated by the projective unitary representation of $\operatorname{Diff}\left(S^{1}\right)$ produces a subnet $\mathcal{A} \subset \mathcal{B}$. This is the Virasoro net having a central charge $c$. We now assume that $c<1$ for our classification. This Virasoro net with $c<1$ falls in the class of coset nets studied by F. Xu $[43,44]$ from the diagonal inclusion $S U(2)_{m-1} \subset S U(2)_{m-2} \otimes S U(2)_{1}$ with $c=1-6 / m(m+1)$, and we know that the Virasoro net $\mathcal{A}$ is completely rational by [29]. We can write down the irreducible representations of the Virasoro nets explicitly and the number of their unitary equivalence classes is $m(m-1) / 2$. We can 
also show that the inclusion $\mathcal{A}(I) \subset \mathcal{B}(I)$ is irreducible, using strong additivity of the Virasoro net that holds by [29], and then a result in [22] implies that the index of $\mathcal{A}(I) \subset \mathcal{B}(I)$ is automatically finite. We can now apply the general machinery of $\alpha$-induction and obtain a modular invariant $Z$ from the inclusion $\mathcal{A}(I) \subset \mathcal{B}(I)$, where the rows and columns of the matrix $Z$ are indexed by the unitary equivalence classes of the irreducible representations of the Virasoro net $\mathcal{A}(I)$. Note that now the dimension of the unitary representation of $S L(2, \mathbb{Z})$ is $m(m-1) / 2$. In this way, we obtain a matrix $Z$ with entries of non-negative integers and dimension $m(m-1) / 2$, starting from a local conformal net $\mathcal{B}$ with diffeomorphism covariance and central charge $c=1-6 / m(m+1), m=2,3,4, \ldots$ This matrix $Z$ is obviously an invariant of the net $\mathcal{B}$ and subject to a strong constraint of modular invariance. The modular invariants of this unitary representation of $S L(2, \mathbb{Z})$ arising from the Virasoro nets have already been classified by Cappelli-Itzykson-Zuber [9] and an explicit classification table is there. The modular invariants are in a bijective correspondence to pairs of Dynkin diagrams whose Coxeter numbers have a difference 1. They are classified into two types. The type I modular invariants are labeled with pairs of Dynkin diagrams $A_{n}, D_{2 n}, E_{6}, E_{8}$, while type II modular invariants with $D_{2 n+1}, E_{7}$ (in pairs with the corresponding $A$-diagrams). (Recall that paragroups of index less than 4 are labeled with Dynkin diagrams $A_{n}, D_{2 n}, E_{6}, E_{8}$ as in $[32,13]$ and appearance of the same diagrams in the context of $S U(2)$ modular invariants is related to locality of the extended nets as in $[1,2,3,4]$.) Locality of the net $\mathcal{B}$ shows that the modular invariant we obtain from $\mathcal{B}$ as above must be of type I. So our classification will be complete if we can show that this map from a net $\mathcal{B}$ to a modular invariant matrix $Z$ of type I in the classification list in [9] is bijective.

This is a problem of classifying irreducible extensions $\mathcal{B} \supset \mathcal{A}$ when the smaller net $\mathcal{A}$ is given. When a modular invariant $Z$ is given and if it arises from an extension $\mathcal{B}$, it is easy to see the decomposition $\bar{\iota} \iota=\bigoplus_{\lambda} Z_{0 \lambda} \lambda$, where $\iota$ is the inclusion map $\mathcal{A}(I) \subset \mathcal{B}(I)$ and $\lambda$ varies in a set of representatives of unitary equivalence classes of irreducible representations of the net $\mathcal{A}$. If we start with a subfactor $\mathcal{A}(I) \subset \mathcal{B}(I)$, then the dual canonical endomorphism $\rho=\bar{\iota} \iota$ and intertwiners $V \in \operatorname{Hom}(\mathrm{id}, \rho)$ and $W \in \operatorname{Hom}\left(\rho, \rho^{2}\right)$ satisfy certain relations. Longo [28] has shown that a triple $(\rho, V, W)$ arising from a subfactor can be axiomatized in terms of simple algebraic relations, and he has called a triple satisfying these relations a $Q$-system. Thus the classification problem is reduced the classification of $Q$-systems $\left(\bigoplus_{\lambda} Z_{0 \lambda} \lambda, V, W\right)$ up to unitary equivalence in a natural sense. A $Q$-system arising from an extension $\mathcal{B} \supset \mathcal{A}$ should also satisfy the chiral locality in [30, Theorem 4.9] in the sense of [3, page 454]. Such a classification problem has been studied for extensions of the $S U(2)_{k}$-nets in several different contexts in $[4,26,33]$. Using such classification results for the $S U(2)_{k^{-}}$ nets, one can also obtain the desired bijectivity for the case of the Virasoro nets, as in [24]. The most subtle step in the $S U(2)_{k}$-classification of the $Q$-systems is uniqueness of the $Q$-system for the $E_{8}$ modular invariant, which was first obtained in [26], and we use this uniqueness result for our classification. Izumi has also made direct computations of this $Q$-system and proved this uniqueness. We also use the 
Böckenahauer-Evans criterion [2] for locality of extensions of the Virasoro nets we construct combinatorially.

The meaning of the labeling in terms of the pairs of Dynkin diagrams in this setting is as follows. Take an example of $\left(A_{10}, E_{6}\right)$. The pair of type $A$ Dynkin diagrams having the same Coxeter numbers is $\left(A_{10}, A_{11}\right)$. This pair of type $A$ diagrams is realized from the Virasoro net with $c=21 / 22$. The representation category of this Virasoro net is a direct product of two systems $A_{10}$ and $A_{11}$ divided by a symmetry of order 2 , in an appropriate sense. (The reason we have a pair of type $A$ diagrams here was clarified in the setting of coset constructions by $\mathrm{Xu}[43,44]$. The number of unitary equivalence classes of irreducible representations is $10 \times 11 / 2=55$.) The representation category of the local conformal net corresponding to the pair $\left(A_{10}, E_{6}\right)$ is a direct product of two systems $A_{10}$ and $A_{3}$ divided by a symmetry of order 2 in the same sense, and the number of unitary equivalence classes of irreducible representations is $10 \times 3 / 2=15$. In this way, the meaning of the label $E_{6}$ is not visible. However, if we use only the $\alpha^{+}$-induction, without $\alpha^{-}$-induction, then we obtain a tensor category of "fake" representations and the system of the irreducible objects is a direct product of two systems $A_{10}$ and $E_{6}$ divided by a symmetry of order 2 in the same sense above, and the number of the irreducible objects is $10 \times 6 / 2=30$. The $E_{6}$ system having six irreducible objects does not arise from a representation category of a net, but it arises from a tensor category of "fake" representations produced with $\alpha^{+}$-induction.

The final classification result in [24] is as follows.

Theorem 3.1. The local conformal nets on the circle with central charge less than 1 are listed as follows.

1. Virasoro nets with central charge $c=1-6 / m(m+1)$.

2. Their simple current extensions of index 2.

3. Four exceptional nets labeled with $\left(A_{10}, E_{6}\right),\left(E_{6}, A_{12}\right),\left(A_{28}, E_{8}\right),\left(E_{8}, A_{30}\right)$.

Two of the exceptional ones in the above list are realized as the coset constructions for $S U(2)_{11} \subset S O(5)_{1} \otimes S U(2)_{1}$ and $S U(2)_{29} \subset\left(G_{2}\right)_{1} \otimes S U(2)_{1}$. Actually, they were considered by Böckenhauer-Evans [1, II, Subsection 5.2] as possible candidates realizing the corresponding modular invariants in the Cappelli-Itzykson-Zuber list, but they were unable to prove that these coset construction indeed produce the desired nets. The other two exceptionals appear to be new.

Note that we did not assume the three conditions for complete rationality in the above, but they automatically hold. This is also analogous to the fact that the (hyperfinite $\mathrm{II}_{1}$ ) subfactors of index less than 4 are automatically amenable. (These subfactors are even of finite depth, actually.) Carpi [8] has recently got a classification for local conformal nets with $c=1$ arising as compact extensions of the Virasoro net.

In the proof of the above theorem, we have used the modular invariants $Z$ arising from local conformal nets as an invariant, but in the statement of the theorem, we 
see that the representation category does gives a complete invariant of such local conformal nets. We also have a complete list of such representation categories. In this way, our goal of the classification we mentioned at the beginning of this paper has been achieved for this class of local conformal nets. It is expected that this type of classification theorem holds in a much wider situation, but proving such a result is beyond the current technique.

We finally remark why this approach based on $\alpha$-induction has worked, in comparison to classification of subfactors. Here, a local conformal net is regarded as an analogue of a subfactor. (They both produce a tensor category through a "representation theory". This gives one analogy.) We have obtained a Virasoro subnet $\mathcal{A}$ from a given net $\mathcal{B}$. In an analogy to subfactor theory in this spirit, the Virasoro subnet corresponds to a subfactor generated by the downward sequence of the Jones projections. That is, for a type $\mathrm{II}_{1}$ subfactor $N \subset M$, we obtain a commuting square

$$
\begin{array}{lll}
N & \subset & M \\
\cup & & \cup \\
P & \subset & Q
\end{array}
$$

where $P$ and $Q$ are generated by the downward sequence of the Jones projections. (Recall that a commuting square is regarded as an embedding of one subfactor into another.) The Virasoro nets are "minimal" in the sense that it is contained in any diffeomorphism covariant net and also in the sense of Carpi's work [7]. Similarly, a subfactor generated by the Jones projections is "minimal" in the sense that it is contained in any subfactor. But the difference is that the Virasoro net is canonically defined while the above subfactor $P \subset Q$ is not canonically determined in $N \subset M$. In this way, we have a control over the $\operatorname{index} \mathcal{A} \subset \mathcal{B}$ while we have no control over the index $P \subset N$. This explains why our approach has worked while a similar approach to classification of subfactors with index less than 4 seems much more difficult (although we have a by far more general classification result for subfactors in [34]).

Acknowledgments. We are grateful to S. Carpi for pointing out inaccuracies in the draft of this paper and R. Longo for detailed comments on it. We also thank J. Fuchs, K.-H. Rehren, C. Schweigert, and the anonymous referee for useful comments.

\section{References}

[1] J. Böckenhauer \& D. E. Evans, Modular invariants, graphs and $\alpha$-induction for nets of subfactors I, Comm. Math. Phys. 197 (1998) 361-386. II 200 (1999) 57-103. III 205 (1999) 183-228.

[2] J. Böckenhauer \& D. E. Evans, Modular invariants from subfactors: Type I coupling matrices and intermediate subfactors, Comm. Math. Phys. 213 (2000) $267-289$. 
[3] J. Böckenhauer, D. E. Evans \& Y. Kawahigashi, On $\alpha$-induction, chiral projectors and modular invariants for subfactors, Comm. Math. Phys. 208 (1999) 429-487.

[4] J. Böckenhauer, D. E. Evans, Y. Kawahigashi, Chiral structure of modular invariants for subfactors, Comm. Math. Phys. 210 (2000) 733-784.

[5] J. Böckenhauer, D. E. Evans \& Y. Kawahigashi, Longo-Rehren subfactors arising from $\alpha$-induction, Publ. Res. Inst. Math. Sci. 31 (2001) 1-35.

[6] D. Buchholz, G. Mack \& I. Todorov, The current algebra on the circle as a germ of local field theories, Nucl. Phys. B, Proc. Suppl. 5B (1988) 20-56.

[7] S. Carpi, Absence of subsystems for the Haag-Kastler net generated by the energymomentum tensor in two-dimensional conformal field theory, Lett. Math. Phys. 45 (1998) 259-267.

[8] S. Carpi, Local extensions of compact type for the $c=1$ Virasoro net, a talk at Miniworkshop "Conformal Field Theory. An Introduction", Rome, March, 2003.

[9] A. Cappelli, C. Itzykson \& J.-B. and Zuber, The A-D-E classification of minimal and $A_{1}^{(1)}$ conformal invariant theories, Comm. Math. Phys. 113 (1987) 1-26.

[10] P. Di Francesco, P. Mathieu \& D. Sénéchal, "Conformal Field Theory", SpringerVerlag, Berlin-Heidelberg-New York, 1996.

[11] S. Doplicher, R. Haag \& J. E. Roberts, Local observables and particle statistics, I. Comm. Math. Phys. 23, 199-230 (1971); II. 35, 49-85 (1974).

[12] S. Doplicher \& J. E. Roberts, A new duality theory for compact groups. Invent. Math. 98 (1989) 157-218

[13] D. E. Evans \& Y. Kawahigashi, "Quantum symmetries on operator algebras", Oxford University Press, 1998.

[14] K. Fredenhagen, K.-H. Rehren \& B. Schroer, Superselection sectors with braid group statistics and exchange algebras II, Rev. Math. Phys. Special issue (1992) $113-157$.

[15] D. Friedan, Z. Qiu \& S. Shenker, Details of the non-unitarity proof for highest weight representations of the Virasoro algebra, Comm. Math. Phys. 107 (1986) $535-542$.

[16] T. Gannon, Modular data: the algebraic combinatorics of conformal field theory, preprint 2001, math.QA/0103044.

[17] P. Goddard, A. Kent \& D. Olive, Unitary representations of the Virasoro and super-Virasoro algebras, Comm. Math. Phys. 103 (1986) 105-119. 
[18] D. Guido \& R. Longo, Relativistic invariance and charge conjugation in quantum field theory, Comm. Math. Phys. 148 (1992) 521-551.

[19] D. Guido \& R. Longo, The conformal spin and statistics theorem, Comm. Math. Phys. 181 (1996) 11-35.

[20] D. Guido, R. Longo \& H.-W. Wiesbrock, Extensions of conformal nets and superselection structures, Comm. Math. Phys. 192 (1998) 217-244.

[21] R. Haag "Local Quantum Physics", Springer-Verlag, Berlin-Heidelberg-New York, (1996).

[22] M. Izumi, R. Longo \& S. Popa A Galois correspondence for compact groups of automorphisms of von Neumann algebras with a generalization to Kac algebras, J. Funct. Anal. 10, (1998) 25-63.

[23] V. F. R. Jones, Index for subfactors, Invent. Math. 72 (1983) 1-25.

[24] Y. Kawahigashi \& R. Longo, Classification of Local Conformal Nets. Case c $<1$, math-ph/0201015, to appear in Ann. Math.

[25] Y. Kawahigashi, R. Longo \& M. Müger, Multi-interval subfactors and modularity of representations in conformal field theory, Comm. Math. Phys. 219 (2001) 631-669.

[26] A. Kirillov Jr. \& V. Ostrik, On q-analog of McKay correspondence and ADE classification of sl ${ }^{(2)}$ conformal field theories, Adv. Math. 171 (2002) 183-227.

[27] R. Longo, Index of subfactors and statistics of quantum fields I-II, Comm. Math. Phys. 126 (1989) 217-247 \& 130 (1990) 285-309.

[28] R. Longo, A duality for Hopf algebras and for subfactors, Comm. Math. Phys. 159 (1994) 133-150.

[29] R. Longo, Conformal subnets and intermediate subfactors, to appear in Comm. Math. Phys., math.OA/0102196.

[30] R. Longo \& K.-H. Rehren, Nets of subfactors, Rev. Math. Phys. 7 (1995) 567597.

[31] R. Longo \& J. E. Roberts, A theory of dimension, K-theory 11 (1997) 103-159.

[32] A. Ocneanu, Quantized group, string algebras and Galois theory for algebras, in Operator algebras and applications, Vol. 2 (Warwick, 1987), (ed. D. E. Evans and M. Takesaki), London Mathematical Society Lecture Note Series 36, Cambridge University Press, Cambridge, 1988, 119-172. 
[33] A. Ocneanu, Paths on Coxeter diagrams: from Platonic solids and singularities to minimal models and subfactors, (Notes recorded by S. Goto), in Lectures on operator theory, (ed. B. V. Rajarama Bhat et al.), The Fields Institute Monographs, AMS Publications, 2000, 243-323.

[34] S. Popa, Classification of amenable subfactors of type II. Acta Math. 172 (1993) 163-255.

[35] K.-H. Rehren, Braid group statistics and their superselection rules, in "The Algebraic Theory of Superselection Sectors", (ed. D. Kastler) World Scientific 1990.

[36] K.-H. Rehren, Chiral observables and modular invariants, Comm. Math. Phys. 208 (2000) 689-712.

[37] V. G. Turaev, "Quantum invariants of knots and 3-manifolds", Walter de Gruyter, Berlin-New York, 1994.

[38] A. Wassermann, Operator algebras and conformal field theory III: Fusion of positive energy representations of $S U(N)$ using bounded operators, Invent. Math. 133 (1998) 467-538.

[39] H.-W. Wiesbrock, Conformal quantum field theory and half-sided modular inclusions of von Neumann algebras, Comm. Math. Phys. 158 (1993) 537-543.

[40] F. Xu, New braided endomorphisms from conformal inclusions, Comm. Math. Phys. 192 (1998) 347-403.

[41] F. Xu, Applications of braided endomorphisms from conformal inclusions, Int. Math. Res. Not. (1998) 5-23.

[42] F. Xu, Jones-Wassermann subfactors for disconnected intervals, Commun. Contemp. Math. 2 (2000) 307-347.

[43] F. Xu, Algebraic coset conformal field theories I, Comm. Math. Phys. 211 (2000) 1-44.

[44] F. Xu, On a conjecture of Kac-Wakimoto, Publ. Res. Inst. Math. Sci. 37 (2001) 165-190.

[45] F. Xu, Algebraic orbifold conformal field theories, in "Mathematical Physics in Mathematics and Physics" (ed. R. Longo), Fields Inst. Commun. 30 (2001), AMS Publications, 429-448.

Added in Proof: After the submission of this paper, the following related two preprints appeared.

S. Köster, Local nature of coset models, preprint 2003, math-ph/0303054. 
F. Xu, Strong additivity and conformal nets, preprint 2003, math.QA/0303266.

In the former, Köster identified one of our two "new" nets, $\left(A_{10}, E_{6}\right)$, with the two cosets $S U(9)_{2} \subset\left(E_{8}\right)_{2}$ and $\left(E_{8}\right)_{3} \subset\left(E_{8}\right)_{2} \otimes\left(E_{8}\right)_{1}$, assuming that the local conformal nets $\left(E_{8}\right)_{k}$ have the expected WZW-fusion rules. The remaining one, $\left(A_{28}, E_{8}\right)$, does not seem to be a coset, and it appears to be a genuine new example.

In the latter, $\mathrm{Xu}$ classified the local conformal nets with $c=1$ with an extra assumption. 